\title{
Análisis del tamaño del gobierno en la economía, perspectiva del consumo y el gasto corriente en el Ecuador 2003 -2013.
}

Analysis of the size of government in the economy, a perspective of consumption and current expenditures in Ecuador 2003 -2013.

Rivera Vargas José Sebastián. ${ }^{1}$,Vargas Ramos María Fernanda.${ }^{2}$,Mayorga Cabrera Fausto Alfredo. ${ }^{3}$ \& Martínez Vaca Patricia Lissette. ${ }^{4}$

DOI: https://doi.org/10.33262/visionariodigital.v3i1.230

\section{Resumen.}

El presente trabajo, es un estudio realizado a partir de las variables Tamaño del Gobierno en la economía, gasto público corriente y el consumo de las familias en Ecuador, desde el año 2003 hasta el 2013, con el fin de analizar la incidencia del tamaño del gobierno en la economía en función de las variables anteriormente mencionadas se procedrá a realizar un modelo econométrico usando el software GRETL.

Con el fin de conocer si el Estado cumple con ciertos parámetros de descentralización estatal se basará el estudio en normativas vigentes, en especial la ratificación de que la descentralización es una herramienta para que los servicios del Estado puedan cubrir la extensión de la Nación, adicionalmente del fomento que genera el Ecuador mediante el tamaño del Estado en la Economía, con especial énfasis en el Gasto Corriente.

${ }^{1}$ Universidad Técnica de Ambato, FCA, Ecuador, j-sebastianrv@hotmail.com

${ }^{2}$ Universidad Tecnológica Indoamérica, Ambato, Ecuador, mariafernandavargas@uti.edu.ec

${ }^{3}$ Universidad Técnica de Ambato, FCA, Ecuador, faustomayorga85@gmail.com ${ }^{4}$ Universidad Técnica de Ambato, FCA, Ambato, Ecuador,patricia-278@hotmail.com 
Palabras Clave: Tamaño del Gobierno en la Economía, Gasto Público Corriente, Consumo de las Familias, Descentralización.

\begin{abstract}
.
The present work is a study made from the variables Size of the Government in the economy, current public expenditure and consumption of families in Ecuador, from 2003 to 2013, in order to analyze the incidence of the size of the Government in the economy based on the aforementioned variables will proceed to make an econometric model using the GRETL software.

In order to know if the State complies with certain parameters of state decentralization, the study will be based on current regulations, especially the ratification that decentralization is a tool for State services to cover the extension of the Nation, in addition to the promotion that Ecuador generates through the size of the State in the Economy, with special emphasis on Current Expenditure.
\end{abstract}

Key Words: Size of the Government in the economy, current public expenditure, consumption of families in Ecuador, Decentralization.

\title{
Introducción.
}

Es garantía del Estado poder brindar servicios para que las instituciones del gobierno proporcionen servicios públicos de salud, educación, energía eléctrica, agua potable y alcantarillado, esto como parte del modelo de descentralización del Estado.

Entre los postulados de la descentralización, según el Plan Nacional de Descentralización se encuentra la cohesión del trabajo del gobierno central y los gobiernos autónomos descentralizados, por tal motivo en el presente documento se analizará la incidencia del tamaño del gobierno en la economía además del consumo de las familias y del gasto público corriente, tomando en cuenta que el acceso equitativo de servicios del Estado, mediante el modelo del traspaso de competencias, permite a la sociedad civil suscribirse al bienestar social.

Ecuador, en su característica política ha demostrado inestabilidad durante el paso de los tiempos, por tanto existen varias tendencias marcadas en las líneas del tiempo de los datos de la economía, en especial en temas de trascendencia del gasto público, es así como estudiaremos diez observaciones de datos en serie de tiempo. 
El motivo principal del estudio es analizar como se comportan ciertas variables que emiten un espectro diferente al de muchos países que promueven su gestión mediante aras del sector privado o la economía real.

A continuación se analizará el comportamiento de las variables mencionadas anteriormente bajo la justificación de cierta normativa que va de la mano de la descentralización.

\section{Conceptualización.}

\section{Consumo De Hogares}

A los gastos en bienes y servicios que realizan los hogares residentes en una economía se conoce como consumo final de hogares. En el enfoque del gasto del Producto Interno Bruto se convierte en uno de los más importantes elementos de la demanda interna.(Chile, 2007)

El ingreso que perciben los hogares y el gasto que realizan los mismos; son elementos centrales para la evaluación y estudio de las condiciones de vida de las familias. (CEPAL, 2011)

\section{Tamaño del gobierno en la economía}

Clasifica las teorías sobre el tamaño y crecimiento del gobierno en dos categorías: del Estado a los ciudadanos y de los ciudadanos al Estado

En la primera categoría del Estado a los ciudadanos, el Estado crece debido a las ineficiencias inherentes en las actividades e incentivos del sector público que enfrentan los burócratas del Gobierno. (Garrett \& Russell M. Rhine, 2006)

(Margarita Katsimi, 1998) Presenta una explicación para el tamaño del sector público basado en la idea de "seguridad social".

- El sector público y el sector privado producen el mismo bien.

- El sector público es menos eficiente versus el privado, en el sentido de que la transferencia de recursos desde el sector privado conduce a una caída en el consumo total.

- El estatus permanente del empleo público en la mayoría de los países, implica una menor volatilidad en la producción pública con respecto al sector privado.

\section{Gasto Público Corriente.}

El gasto corriente se refiere a la adquisición de bienes y servicios que realiza el gobierno en un periodo fiscal sin incrementar el patrimonio, ejemplo: sueldos y salarios de servidores públicos. 
Tanto gastos como ingresos del sector público se han expandido en los últimos años de la mano del crecimiento económico. Sin embargo, a partir del año 2007 el crecimiento del gasto se incrementa sustancialmente en materia de la recuperación del rol del Estado. (Oleas, Mosquera, \& Bucaram, 2015)

Este tipo de gasto incluye las erogaciones necesarias para que las instituciones del gobierno proporcionen servicios públicos de salud, educación, energía eléctrica, agua potable y alcantarillado, entre otros, así como para cubrir el pago de las pensiones y los subsidios destinados a elevar el bienestar de la población de menores ingresos. (México, 2008)

Aquí están inmersos; subsidios para los planes de desarrollo urbano, rural, implementación de enseres farmacéuticos y además de sueldos a educación, seguridad, salud, etc.

El gasto corriente se refiere a la adquisición de bienes y servicios que realiza el sector público durante el ejercicio fiscal sin incrementar el patrimonio del estado. Los gastos corrientes pueden descomponerse según el tipo de gasto en sueldos y salarios, compras de bienes y servicios, pagos de intereses, subsidios y transferencias corrientes y otros gastos corrientes. (Fondo Monetario Internacional, 2007)

Sin embargo para Turnovsky y Fisher, (1995) plantean una diferencia entre los gastos del gobierno que aportan en un mejoramiento de la utilidad y los que afectan en la operación de las empresas, concluye su planteamiento "el gasto público se destina en beneficio de los consumidores, prevalecen del sector privado" este supuesto afirma que esta actividad afecta a los recursos disponibles del sector privado.

\section{Justificación.}

El déficit estructural que afecta a las finanzas públicas ecuatorianas se origina mucho antes de la caída del precio del crudo en el 2014. Esta reducción a menos de la mitad de lo que era en 2014, exacerba una situación fiscal complicada que en los últimos tiempos se ha caracterizado por un gasto público que consistentemente excede a los ingresos. (Oleas, Mosquera, \& Bucaram, 2015)

El gasto del consumo de los hogares en el Ecuador desde la dolarización como porcentaje del PIB ha venido comportándose de una manera estable, dado la poca volatilidad de esta variable que básicamente depende de los tipos de interés real, de las expectativas de empleo futuro y de la riqueza de los individuos. Desde el año 2000 hasta el segundo trimestre del año 2015 el consumo de los hogares ha participado en el PIB en un 64\% en media. (Camino, 2015) 
El nuevo plan de desarrollo, el "Socialismo del Buen Vivir", se fundamenta en la enorme magnitud y eficiencia del gasto público y hace del mismo y del papel del Estado en la economía, las fuerzas motrices para relanzar el proyecto de construcción económica y social que ubique al Ecuador de cara a la industrialización del actual milenio. (Arévalo, 2014)

Con el fin de analizar la propuesta econométrica y darle valor agregado al estudio de la descentralización del Estado, el presente documento se articula a las normativas mencionadas en la introducción, dicha normativa tiene base en la COOTAD.

COOTAD.- Código orgánico de organización territorial, autonomía y descentralización.

Art. 1.- Ámbito.- Este Código establece la organización político-administrativa del Estado ecuatoriano en el territorio: el régimen de los diferentes niveles de gobiernos autónomos descentralizados y los regímenes especiales, con el fin de garantizar su autonomía política, administrativa y financiera. Además, desarrolla un modelo de descentralización obligatoria y progresiva a través del sistema nacional de competencias, la institucionalidad responsable de su administración, las fuentes de financiamiento y la definición de políticas y mecanismos para compensar los desequilibrios en el desarrollo territorial. (Descentralización, 2012) (Competencias, 2010)

\section{Concordancias:}

CONSTITUCION DE LA REPUBLICA DEL ECUADOR, Arts. 4, 238, 239

Art. 2.- Objetivos.- Son objetivos del presente Código:

- La profundización del proceso de autonomías y descentralización del Estado, con el fin de promover el desarrollo equitativo, solidario y sustentable del territorio, la integración y participación ciudadana, así como el desarrollo social y económico de la población;

- El fortalecimiento del rol del Estado mediante la consolidación de cada uno de sus niveles de gobierno, en la administración de sus circunscripciones territoriales, con el fin de impulsar el desarrollo nacional y garantizar el pleno ejercicio de los derechos sin discriminación alguna, así como la prestación adecuada de los servicios públicos;

- La democratización de la gestión del gobierno central y de los gobiernos autónomos descentralizados, mediante el impulso de la participación ciudadana;

- La definición de mecanismos de articulación, coordinación y corresponsabilidad entre los distintos niveles de gobierno para una adecuada planificación y gestión pública; 
- La distribución de los recursos en los distintos niveles de gobierno, conforme con los criterios establecidos en la Constitución de la República para garantizar su uso eficiente.

Concordancias:

• CONSTITUCIÓN DE LA REPÚBLICA DEL ECUADOR, Arts. 60, 238, 239

\section{Metodología.}

Con el fin de estudiar los enfoques de la descentralización del Estado; se aplicó un modelo econométrico, el cual, con base en la justificación, permitirá conocer la incidencia del Tamaño del Estado en la Economía en función del Consumo de las Familias y el Gasto Público Corriente.

Bajo esta premisa, se explicará en primer lugar el modelo matemático econométrico.

Datos.

\begin{tabular}{|c|c|c|c|}
\hline \multirow[t]{6}{*}{ Años } & Tamaño del & Gasto & Gasto Público \\
\hline & Gobierno en la & consumo & \multirow{5}{*}{$\begin{array}{l}\text { Corriente } \\
\text { (Miles USD) }\end{array}$} \\
\hline & economía & hogares & \\
\hline & Miles USD & Residentes & \\
\hline & & (Miles & \\
\hline & & dólares 2007) & \\
\hline 2003 & 32158291 & 27461589 & 1727,776445 \\
\hline 2004 & 34136114 & 29228969 & 2342,887392 \\
\hline 2005 & 35604531 & 30523141 & 3192,134437 \\
\hline 2006 & 37125765 & 31851533 & 3755,684765 \\
\hline 2007 & 38775487 & 33201278 & 6205,022404 \\
\hline 2008 & 41186204 & 34994856 & 9343,9047 \\
\hline 2009 & 41558650 & 34648396 & 7148,006803 \\
\hline 2010 & 44534140 & 37320635 & 9283,943566 \\
\hline 2011 & 47075511 & 39234629 & 12582,62691 \\
\hline 2012 & 49010047 & 40297955 & 13809,03645 \\
\hline 2013 & 50967555 & 41582257 & 14974,84849 \\
\hline
\end{tabular}

Fuente: Banco Central del Ecuador 
Realizado por: Grupo Investigador
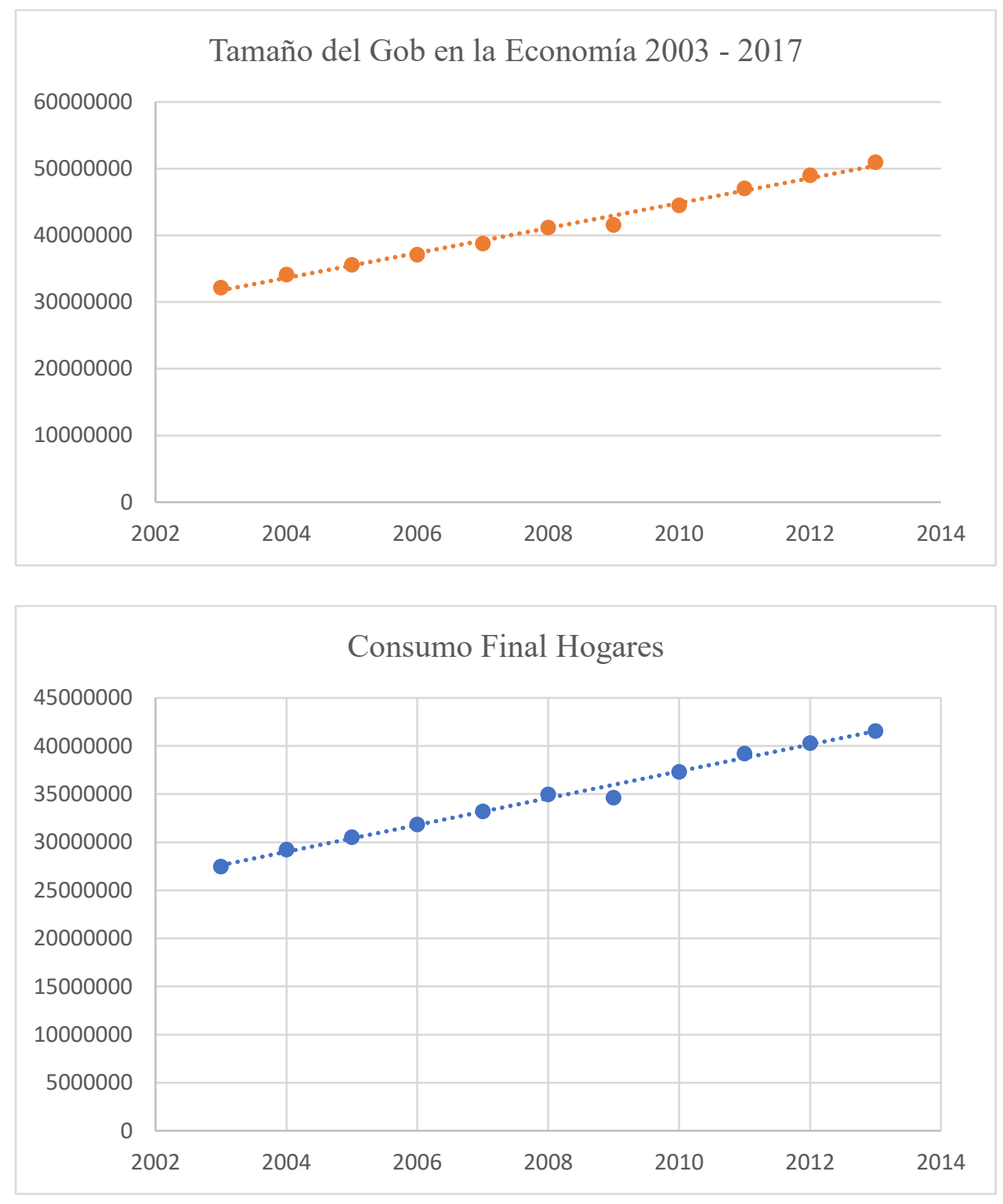

Fuente: Banco Central del Ecuador

Realizado por: Grupo Investigador 


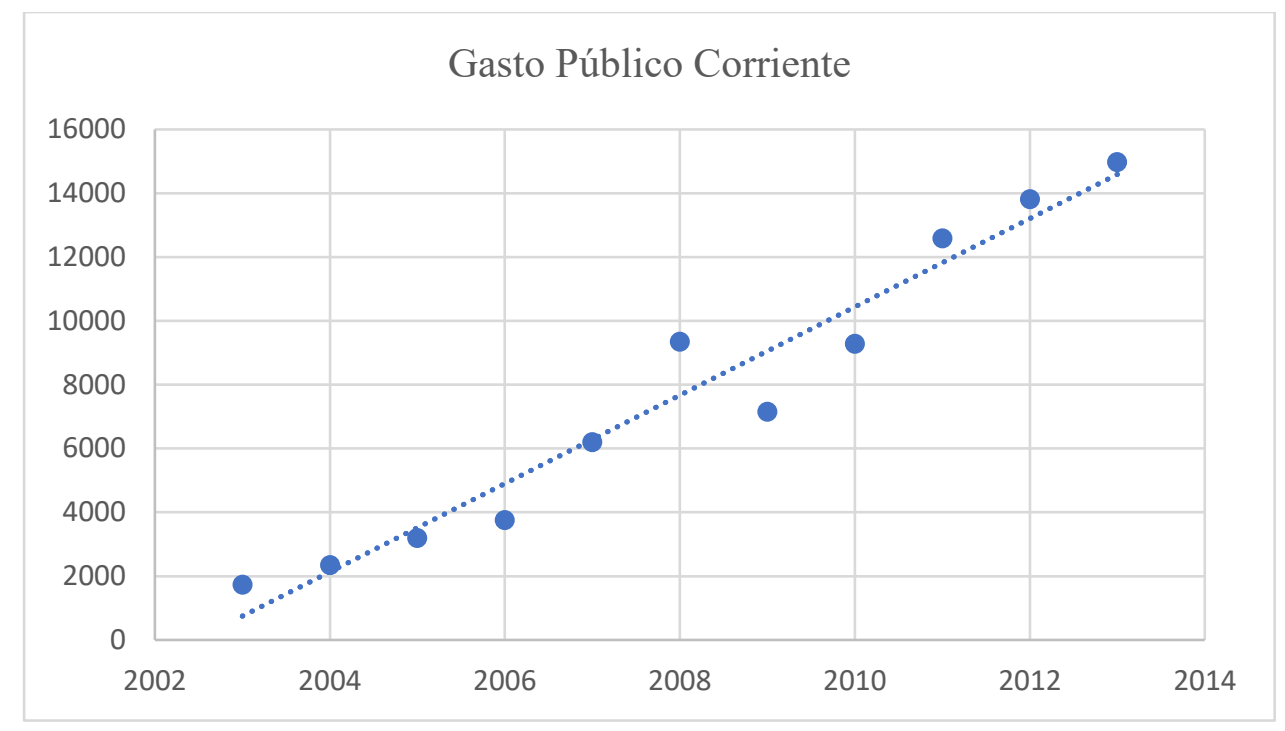

Fuente: Banco Central del Ecuador

Realizado por: Grupo Investigador

- Modelo Econométrico

Ecuasión:

$$
y=\beta_{0}+\beta_{1} x_{1}+\beta_{2} x_{2}+u
$$

Donde;

$\mathrm{Y}=$ Tamaño del Gobierno en la Economía

$\beta_{0}=$ Valor de la Constante.

$\mathrm{X} 1=$ Consumo de las Familias.

$\mathrm{X} 2=$ Gasto Público Corriente .

$u=$ Error

Para el desarrollo del modelo ya explicado, se usó el software GRETL, aplicando un modelo de mínimo cuadrados ordinario con un total de 10 observaciones. Cabe mencionar que con el fin de evitar errores seriales se aplicó una diferencia logarítmica a la variable dependiente y al regresor 1 .

Obteniendo los siguientes resultados: 


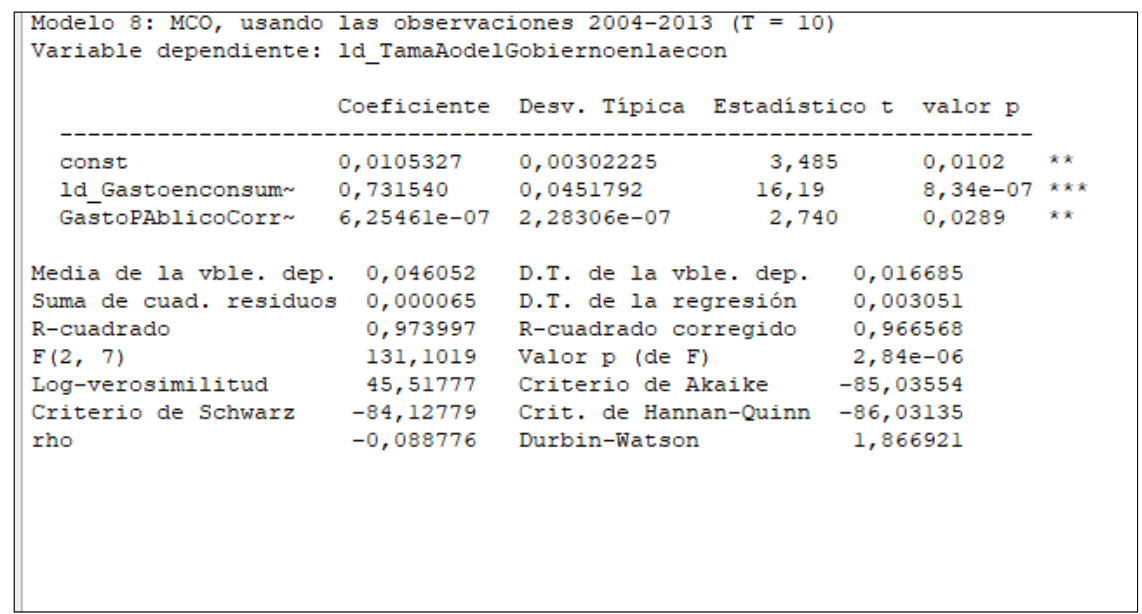

Fuente: Gretl

Realizado por: Grupo Investigador

En la imagen se pueden observar las siguientes apreciaciones respecto a la significatividad del modelo:

- En primera instancia, los valores de desviación típica no son altos

- Los valores absolutos del estadístico t, son mayores a 2.

- El R-cuadrado responde a un 0,97 , lo que indica un $97 \%$ de correlación entre las variables.

- Dado, que se procedió a añadir diferencias logarítmicas a las variables dependiente y al primer Regresor; se denota la eliminación de tendencias en la línea de tiempo.

- Se nota como resultado del ítem anterior que no existe autocorrelación o error serial pues el estadístico Durbin Watson está en un valor a 2.

- El Estadístico de Fisher nos da un valor medianamente alto.

Adicionalmente se realizan las siguientes pruebas para comprobar contraste: 


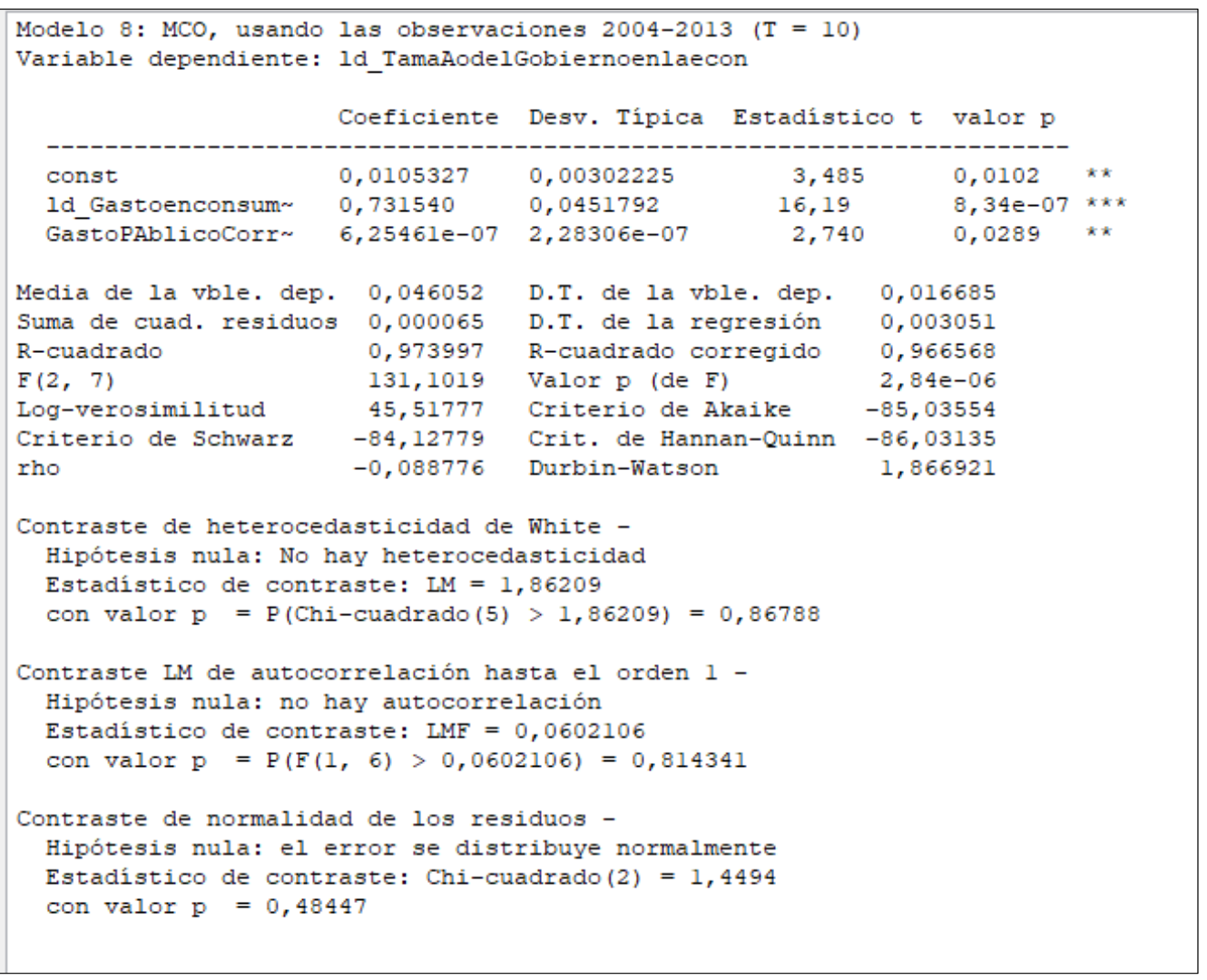

Fuente: Gretl

Realizado por: Grupo Investigador 


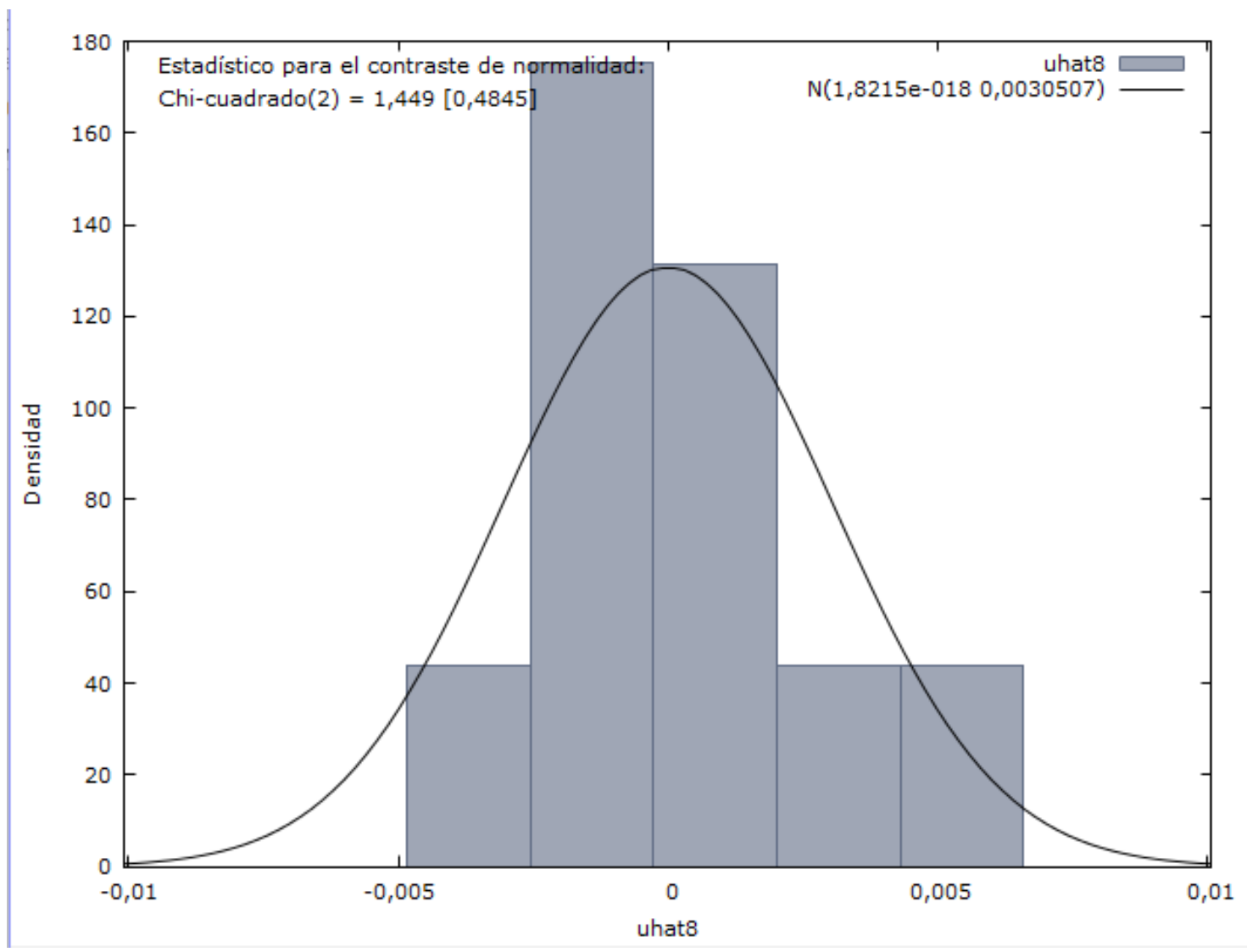

Fuente: Gretl

Realizado por: Grupo Investigador

En la última gráfica se puede apreciar el contraste de normalidad de los errores.

\section{Resultados.}

Una vez aplicado el modelo econométrico se obtienen los siguientes resultados:

- Corte en “y” (constante) $=0,0105327$

- Consumo Familias $=0,731540$

- Gasto Corriente $=6,25 \mathrm{e}^{\wedge}-07$

Lo que significa:

Cada vez que el Tamaño del Gobierno en la economía en 0,011 unidades, el consumo de las familias aumenta en 0,73 y el gasto corriente en 6,25 e-07. 


\section{Conclusiones.}

- El modelo econométrico aplicado tiene fuerte significatividad.

- Se comprueba que, con base a la justificación del documento, el Estado se encuentra impulsando y fomentando productividad la misma que se refleja en consumo de las familias.

- El modelo de descentralización, mediante el traspaso de poderes, busca el acceso inclusivo y cohesionado para la población, mismo que puede medirse por el tamaño del gobierno en la economía.

- El modelo de la amplitud del Estado en la Economía, permite el aumento de ingresos per cápita, que deriva en consumo aumentando ingresos fiscales a las arcas del país.

\section{Bibliografía}

Ahumada, G. (1992). Tratado de finanzas Publicas (4a ed.). Buenos Aires: Plus Ultra.

CEPAL. (Enero de 2011). Ingresos y gastos de consumo de hogares. Obtenido de https://repositorio.cepal.org/bitstream/handle/11362/4718/S01010054_es.pdf?sequenc e

Chile, B. C. (2007). Consumo de Hogares . Obtenido de https://si3.bcentral.cl/estadisticas/Principal1/Metodologias/CCNN/anuales/Consumo_ Hogares.pdf

Competencias, C. N. (2010). COOTAD. Quito.

Descentralización, C. O. (2012). COOTAD. COOTAD. Ecuador.

Docencia Economia Española. (2000). Renta Nacional Bruta. España: Jarriola.

Fondo Monetario Internacional. (1 de Febrero de 2007). Public Expenditure in Latin America : Trends and Key Policy Issues. Obtenido de http://www.imf.org/en/Publications/WP/Issues/2016/12/31/Public-Expenditure-inLatin-America-Trends-and-Key-Policy-Issues-20244

Garrett, T. A., \& Russell M. Rhine. (2006). Russell M. Rhine. Obtenido de https://files.stlouisfed.org/files/htdocs/publications/review/06/01/GarrettRhine.pdf

Griziotti, B. (1939). Principios de la cinecia de las finanzas. Buenos Aires: Depalma. 
Margarita Katsimi. (Julio de 1998). Explaining the Size of the Public Sector. Obtenido de https://www.jstor.org/stable/30024381

México, S. d. (2008). Secrétaría de Hacienda México. Obtenido de http://www.apartados.hacienda.gob.mx/presupuesto/temas/ppef/2006/temas/expo_mot ivos/em02.pdf

Oleas, S., Mosquera, S., \& Bucaram, S. (Octubre de 2015). La República EC. Obtenido de https://www.larepublica.ec/blog/opinion/2015/10/16/situacion-fiscal-ecuador/

Panorama Internacional. (2009). Producto Interno Bruto per cápita (2007). Panorama Educativo de México 2009, CS07.

Turnovsky, S., \& W., F. (1995). The composition of government expenditure and its consequences for macroeconomic performance. Journal of economics Dynamics nd control, 747-786.

William A. Niskanen. (1971-2001). The Elgar Companion to Public Choice. Obtenido de https://www.elgaronline.com/view/1852785403.00023.xml

\section{Para citar el artículo indexado.}

Rivera J, Vargas M, Mayorga F. \& Martínez L. (2018). Análisis del tamaño del gobierno en la economía, perspectiva del consumo y el gasto corriente en el Ecuador 2003 -2013.Revista electrónica Visionario Digital 1(1), 52-66. Recuperado desde: http://cienciadigital.org/revistacienciadigital2/index.php/VisionarioDigital/article/view/230/5 05

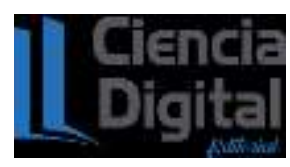

El artículo que se publica es de exclusiva responsabilidad de los autores y no necesariamente reflejan el pensamiento de la Revista Ciencia Digital.

El articulo queda en propiedad de la revista y, por tanto, su publicación parcial y/o total en otro medio tiene que ser autorizado por el director de la Revista Ciencia Digital



